\title{
Descrição dos pupários de Manotes crassimanus James e Pedinocera longicornis Kertész (Diptera, Stratiomyidae, Pachygastrinae) da Ilha da Marambaia, Mangaratiba, Rio de Janeiro, Brasil
}

\author{
Ana L. de F. Lopes ${ }^{1}$; Roberto de Xerez ${ }^{1}$; Roberta R. da Silva ${ }^{1} \&$ José R. Pujol-Luz ${ }^{2}$ \\ ${ }^{1}$ Laboratório de Biologia e Ecologia de Diptera, Departamento de Biologia Animal, Instituto de Biologia, Universidade Federal \\ Rural do Rio de Janeiro. Rodovia BR-465, km 07, 23890-000 Seropédica, Rio de Janeiro, Brasil. E-mail: \\ anaflopes@globo.com; rdexerez@ufrrj.br; betaufrrj@yahoo.com.br. \\ 2 Departamento de Zoologia, Instituto de Biologia, Universidade de Brasília. 70910-900 Brasília, Distrito Federal, Brasil. E- \\ mail: jrpujol@unb.br
}

\begin{abstract}
Description of the puparia of Manotes crassimanus James and Pedinocera longicornis Kertész (Diptera, Stratiomyidae, Pachygastrinae) from Ilha da Marambaia, Mangaratiba, Rio de Janeiro, Brazil. Two larvae of Manotes crassimanus and 12 larvae of Pedinocera longicornis were collected under the bark of fallen trees at Ilha da Marambaia $\left(23^{\circ} 04^{\prime} 15^{\prime \prime} \mathrm{S}, 43^{\circ} 53^{\prime} 59^{\prime \prime} \mathrm{W}\right.$, sea level), Mangaratiba, State of Rio de Janeiro, Brazil. After the emergence of adult, the puparia of those species were described for the first time and compared with other species of Pachygastrinae.

KEY WORDS. Decomposition, larvae, Neotropical, taxonomy.
\end{abstract}

RESUMO. Duas larvas de Manotes crassimanus e 12 de Pedinocera longicornis foram coletadas sob a casca de árvores

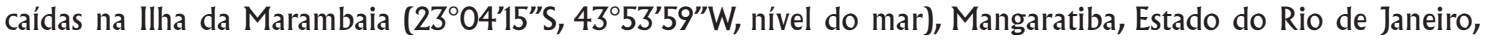
Brasil. Após a emergência do adulto, os pupários destas espécies foram descritos pela primeira vez e comparados com o de outras espécies de Pachygastrinae.

PALAVRAS-CHAVE. Decomposição, larvas, Neotropical, taxonomia.

Stratiomyidae reúne 12 subfamílias com 375 gêneros e 2651 espécies. Na região Neotropical são reconhecidas 987 espécies (Woodley 2001). Ocorrem em regiões tropicais e temperadas do mundo, porém são mais comuns em climas tropicais chuvosos. Adultos são freqüentemente encontrados em restos vegetais, visitando flores ou frutos caídos. Suas larvas podem ser encontradas em ambientes terrestres ou aquáticos e, eventualmente, em substâncias orgânicas de origem animal ou vegetal em decomposição (Brindle 1963, McFadden 1967, James 1973, RozkošnÝ 1982, WoOdLeY 2001).

Larvas de Pachygastrinae têm características terrestres sendo comumente encontradas sob casca de árvore em decomposição, mas algumas vezes também nas raízes de plantas. Podem ser reconhecidas pelos seguintes caracteres: (1) lobos oculares ausentes, (2) cerdas $\mathrm{Cf}_{2}$ e V2 situadas na frente dos olhos, (3) cerdas sublaterais ausentes; (4) 5 cerdas ventrais sobre o segmento anal (RoZKošNÝ 1982).

Das 136 espécies de Pachygastrinae conhecidas na região Neotropical (Woodley 2001), são conhecidas larvas ou pupários de apenas nove espécies: Chalcidomorphina aurata Enderlein, 1914, Cosmariomyia argyrosticta Kertèsz, 1914, Dactylodeictes lopesi
Lindner, 1964, Engicerus major Lindner, 1964, Eupachygaster alexanderi (James, 1967) Popanomyia femoralis Kertèsz, 1909, Psephiocera modesta (Lindner, 1949), Vittiger schnusei Kertèsz, 1909 e Zabrachia stoichoides James, 1965, descrições estas feitas respectivamente por: Pujol-Luz \& Xerez (1999); Xerez et al. (2002) (C. argyrosticta e D. lopesi); Xerez et al. (2003a) (E. major e $P$. femoralis); Blanchard (1922); Xerez et al. (2003b); Xerez \& PujolLuz (2001) e JAMEs (1965).

Neste trabalho são descritos os pupários de Manotes crassimanus James, 1980 e Pedinocera longicornis Kertèsz, 1909 e comparados com os pupários já descritos de sete outras espécies de Pachygastrinae.

\section{MATERIAL E MÉTODOS}

Foram coletadas duas larvas de Manotes crassimanus e 12 de Pedinocera longicornis sob casca de troncos de árvores em estágio inicial de decomposição, na Ilha da Marambaia (2304'15"S, 4353'59"W, nível do mar), Mangaratiba, Estado do Rio de Janeiro. As larvas foram acondicionadas em placas de Petri junto com o substrato coletado, seguindo a metodologia para criação Pujol-Luz \& Xerez (1999), Xerez \& Pujol-Luz (2001)

Revista Brasileira de Zoologia 23 (3): 733-739, setembro, 2006 
e XEREz et al. (2002). Após a emergência, os adultos foram montados em alfinetes entomológicos e os pupários foram fixados em microvails em uma solução 3:1 de álcool a 70\% e glicerol. Os pupários foram observados e desenhados em estereomicroscópio com câmara clara aclopada. Para as fotografias foi utilizada máquina fotográfica digital. Fotos das cerdas torácicas e da mancha esternal de $P$. longicornis foram feitas no microscópio eletrônico de varredura (MEV) da Empresa Brasileira de Pesquisa Agropecuária - Agrobiologia. A larva foi desidratada até o ponto crítico e metalizada, de acordo com as técnicas de rotina para MEV. A identificação foi feita com base na morfologia de adultos, uma vez que a chave de identificação de larvas existente não abrange todas as espécies de Pachygastrinae neotropicais. Os espécimes foram incorporados à Coleção Entomológica Costa Lima (CECL), Universidade Federal Rural do Rio de Janeiro, Seropédica, Rio de Janeiro, Brasil. A terminologia adotada na descrição segue a de JAMEs (1981) e RoskošnÝ \& KovaC (1994).

\section{RESULTADOS}

\section{Manotes crassimanus James, 1980 Figs 1-11}

Registros de distribuição. Brasil (nova ocorrência): Rio de Janeiro, Ilha da Marambaia; México: Morelos, Cuernavaca.

Pupário. Comprimento: 5,6 mm. Pupário achatado dorsoventralmente (Fig. 11), margens laterais dos segmentos do corpo fortemente arqueadas. Cutícula com aparência usual de mosaico, algumas células formando manchas características e placas em todos os segmentos torácicos e abdominais, dorso e ventralmente. Cerdas de todos os segmentos torácicos e abdominais plumosas; cerdas dorsais do primeiro segmento torácico claviformes; cerdas dorsolaterais da cabeça plumosas e claviformes (Fig. 1). Padrão cromático amarelo claro.

Cabeça. Triangular, achatada dorsoventralmente (Fig. 1), comprimento maior que a largura (Fig. 2), complexo mandibulomaxila bem desenvolvido, escovas cilíndricas quase tão longas quanto o labro em vista dorsal; labro triangular; antenas curtas, bissegmentadas, surgindo na parte anterior da cabeça. Olhos pouco proeminentes, arredondados, situados na parte posterior da cabeça; linhas craniais dorsais fortemente invaginadas. Quetotaxia: dois pares de cerdas clipeofrontais, um par de cerdas dorsolateral inserido acima dos olhos, um par de cerdas lateral inserido abaixo dos olhos, três pares de cerdas ventrolaterais e três pares de cerdas ventrais em forma de espinho (Figs 1, 2 e 8).

Tórax. Primeiro segmento com duas fileiras de cerdas; dois pares de cerdas anterodorsais e três pares de cerdas dorsais; um par de cerdas dorsolateral (Fig. 1) e um par de cerdas ventrolateral inseridos acima do espiráculo; dois pares de cerdas ventrais, sendo o par mais externo maior e bifurcado (Fig. 2). Segundo e terceiro segmentos com três pares de cerdas dorsais quase tão longas quanto as laterais, um par de cerdas lateral (Fig. 1), um par de cerdas ventrolateral, dois pares de cerdas ventrais, sendo o mais externo maior e bifurcado (Fig. 2).
Abdômen. Primeiro ao sétimo segmento com a mesma forma, apresentando três pares de cerdas dorsais decrescendo de tamanho do mais interno para o mais externo, um par de cerdas dorsolateral, um par de cerdas lateral (Fig. 3); dois pares de cerdas ventrolaterais e três pares de cerdas ventrais decrescendo de tamanho do mais externo para o mais interno; linha ventromediana do sexto segmento com mancha elíptica dilatada na região voltada para a parte anterior do corpo (Figs 4 e 10); espiráculo respiratório pupal presente somente nos segmentos um a seis (Fig. 3); oitavo segmento com um par de cerdas dorsocentral, dois pares de cerdas laterais, um par de cerdas subapical, um par de cerdas apical e cinco pares de cerdas ventrais (Figs 3 e 4), cerda $\mathrm{V}_{2}$ muito pequena, assemelhando-se a um pêlo.

Material examinado. Brasil, Rio de Janeiro: Ilha da Marambaia, 16/I/2003, G.G. Viana, R. da Silva, K. Marques leg., 2 pupários, 2 adultos (emergências: 10/III/2003, 07/IV/2003).

\section{Pedinocera longicornis Kertèsz, 1909 Figs 12-22}

Registro de distribuição. Brasil: Rio de Janeiro (nova ocorrência), Ilha da Marambaia; Peru: Ucayali River, Unini.

Pupário. Comprimento 5,6 a 7,1 mm, achatado dorsoventralmente, margens laterais dos segmentos do corpo fortemente arqueadas (Fig. 21). Cutícula com usual aparência de mosaico, algumas células formando manchas características nas regiões ventral e dorsal. Padrão cromático marrom-amarelado claro.

Cabeça. Triangular, achatada dorsoventralmente, comprimento maior que a largura; complexo mandíbulo-maxila bem desenvolvido, escovas cilíndricas quase tão longas quanto o labro em vista dorsal; labro triangular. Antenas curtas, bissegmentadas surgindo na parte anterior da cabeça. Olhos pouco proeminentes, arredondados aparecendo na parte posterior da cabeça. Quetotaxia: dois pares de cerdas clipeofrontais, um par de cerdas dorsolateral inserido acima dos olhos, um par de cerdas lateral inserido abaixo dos olhos, três pares de cerdas ventrolaterais e três pares de cerdas ventrais em forma de espinho (Figs 12, 13 e 18).

Tórax. Primeiro segmento menor que os outros com duas fileiras de cerdas dorsais; dois pares de cerdas anterodorsais com o par mais externo maior, e três pares de cerdas dorsais; um par de cerdas dorsolateral (Figs 12 e 20) e um par de cerdas ventrolateral inseridos acima do espiráculo; dois pares de cerdas ventrais, sendo o mais externo maior e bifurcado (Figs 13 e 19). Segundo e terceiro segmentos com uma fileira de cerdas, sendo três pares de cerdas dorsais, decrescendo do mais interno para o mais externo, um par de cerdas lateral, um par de cerdas ventrolateral e dois pares de cerdas ventrais, sendo o mais externo maior e bifurcado (Figs 12 e 13).

Abdômen. Segmentos de 1-7 com o mesmo formato (Fig. 21), com uma fileira de cerdas, sendo três pares de cerdas dorsais, decrescendo de tamanho do mais interno para o mais externo, um par de cerdas dorsolateral, um par de cerdas lateral, dois pares de cerdas ventrolaterais e três pares de cerdas ventrais do 

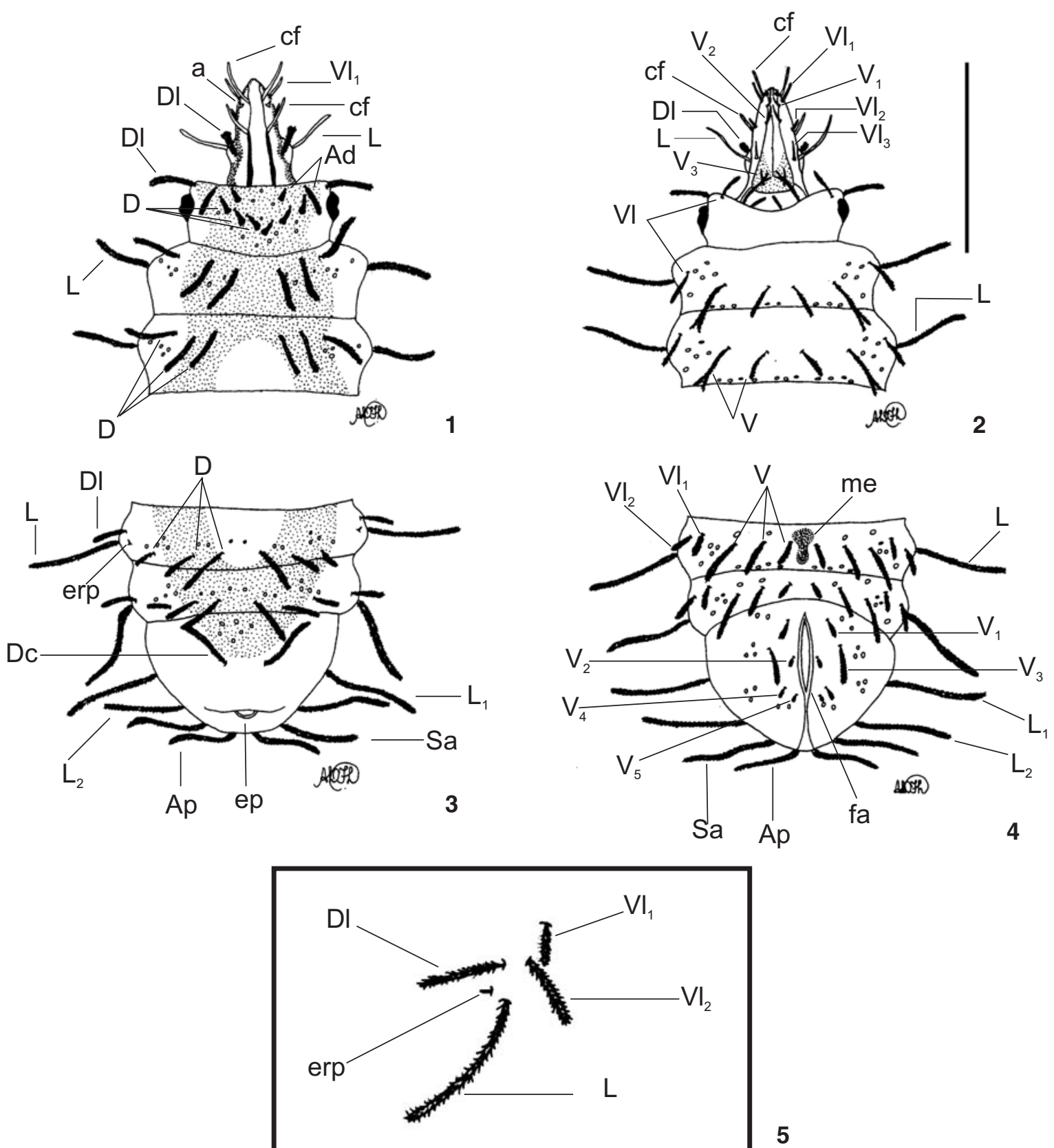

5

Figuras 1-5. Manotes crassimanus. (1) cabeça: $1^{\circ}, 2^{\circ}$ e $3^{\circ}$ segmentos torácicos (vista dorsal); (2) Cabeça: $1^{\circ}, 2^{\circ}$ e $3^{\circ}$ segmentos torácicos (vista ventral); (3) $6^{\circ}, 7^{\circ}$ e $8^{\circ}$ segmentos abdominais (vista dorsal); (4) $6^{\circ}, 7^{\circ}$ e $8^{\circ}$ segmentos abdominais (vista ventral); (5) esquema das cerdas da região lateral dos segmentos abdominais. (a) antena; (Ad) cerdas anterodorsais; (Ap) cerdas apicais; (cf) cerdas clipeofrontais; (D) cerdas dorsais; (Dc) cerdas dorsocentrais; (DI) cerdas dorsolaterais; (ep) espiráculo posterior; (erp) espiráculo respiratório pupal; (fa) fenda anal; (L) cerdas laterais; (me) mancha esternal; (Sa) cerdas subapicais; (V) cerdas ventrais; (Vl) cerdas ventrolaterais. Barra de escala = $1 \mathrm{~mm}$ (Figs 1 a 4 ).

mesmo tamanho (Figs 14 e 15); linha ventromediana do sexto segmento com mancha elíptica estreitada levemente no meio (Figs 15 e 22); espiráculo respiratório pupal presente do pri- meiro ao sexto segmento abdominal (Fig. 16). Segmento $8 \mathrm{com}$ um par de cerdas dorsocentrais, dois pares de cerdas laterais, um par de cerdas subapical, um par de cerdas apical e cinco

Revista Brasileira de Zoologia 23 (3): 733-739, setembro, 2006 

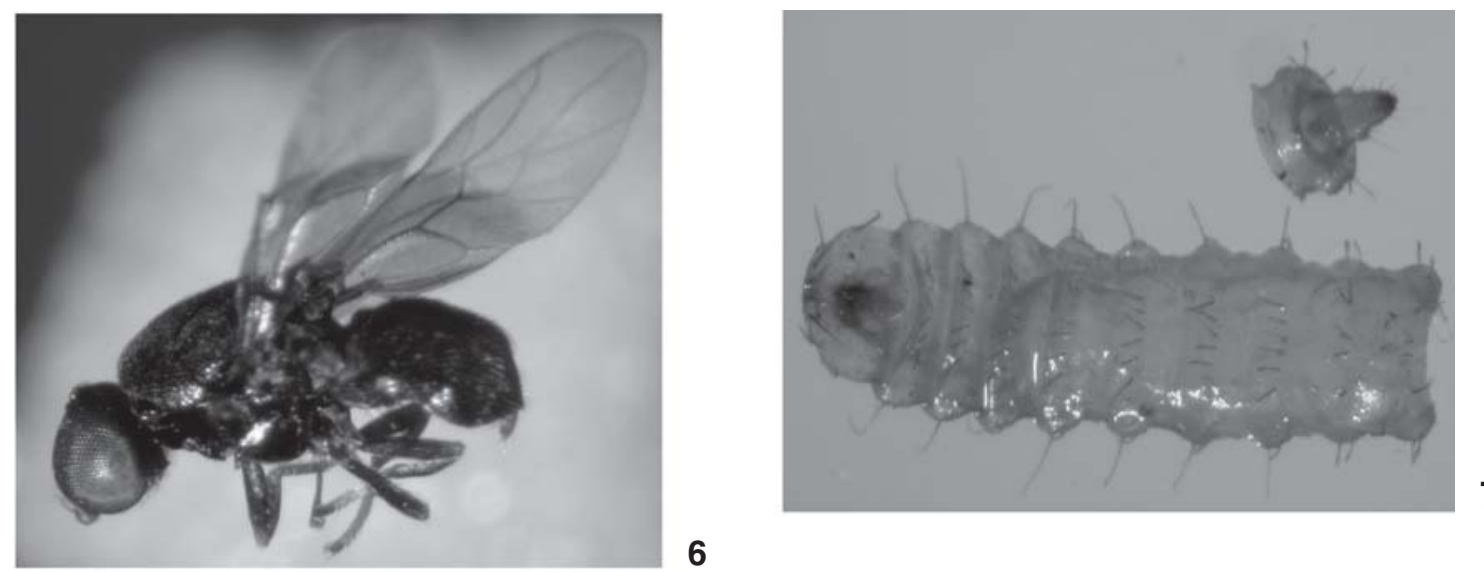

\section{6}
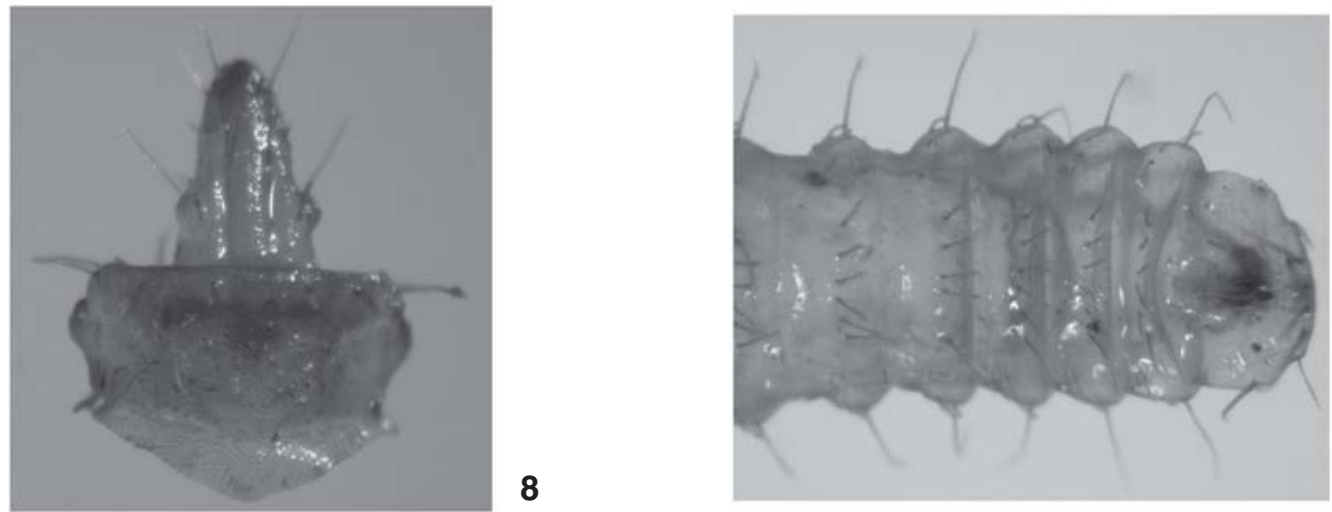

9
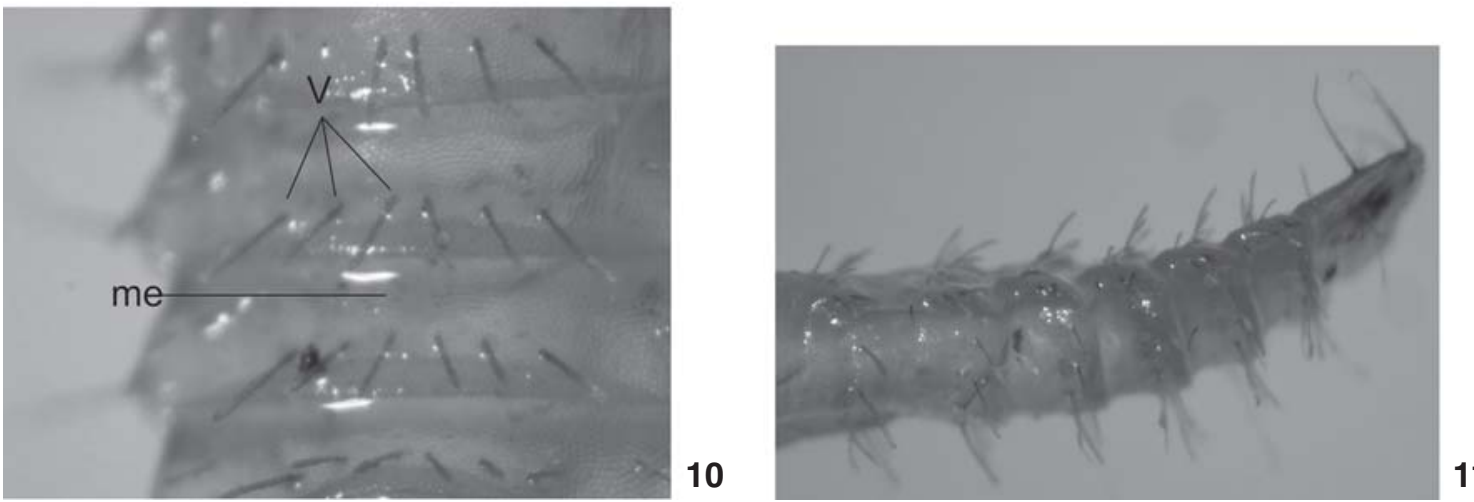

Figuras 6-11 Manotes crassimanus: (6) adulto; (7) pupário; (8) detalhe da cabeça e $1^{\circ}$ segmento torácico; (9) detalhe dos segmentos 2 8 abdominais; (10) detalhe do $5^{\circ}$ e $6^{\circ}$ segmento abdominal; (11) visão lateral do pupário. (me) mancha esternal; (V) cerdas ventrais.

pares de cerdas ventrais (Figs 14 e 15)

Material examinado. BRASIL, Rio de Janeiro: Ilha da Marambaia, 02/X/2000, 03/II/2003, 10/II/2003 e 17/II/2003, R. de Xerez, G.G. Viana, R.R. da Silva, K.I.S. Marques, A.L.F. Lopes, M.M.O. Sant'Anna, L.P. D'Almeida leg., 12 pupários, 65 adultos (emergências: 02/X/2000, 29/I/2003, 31/I/2003, 03-07/II/2003, 10/II/2003, 12/II/2003, 17/II/2003, 18/II/2003, 24/II/2003, 25/ II/2003, 28/II/2003, 10/III/2003).

\section{DISCUSSÃO}

Foi observado em M. crassimanus e em P. longicornis, assim como em pupários das sete espécies estudadas, C. aurata; $V$. schnusei; C. argyrosticta; D. lopesi; P. femoralis; E. major e P. modesta a presença, próximo à emergência, de uma linha transversal entre o primeiro e o segundo segmentos torácicos e de uma linha dorso-mediana a partir do segundo segmento torácico atin-

Revista Brasileira de Zoologia 23 (3): 733-739, setembro, 2006 

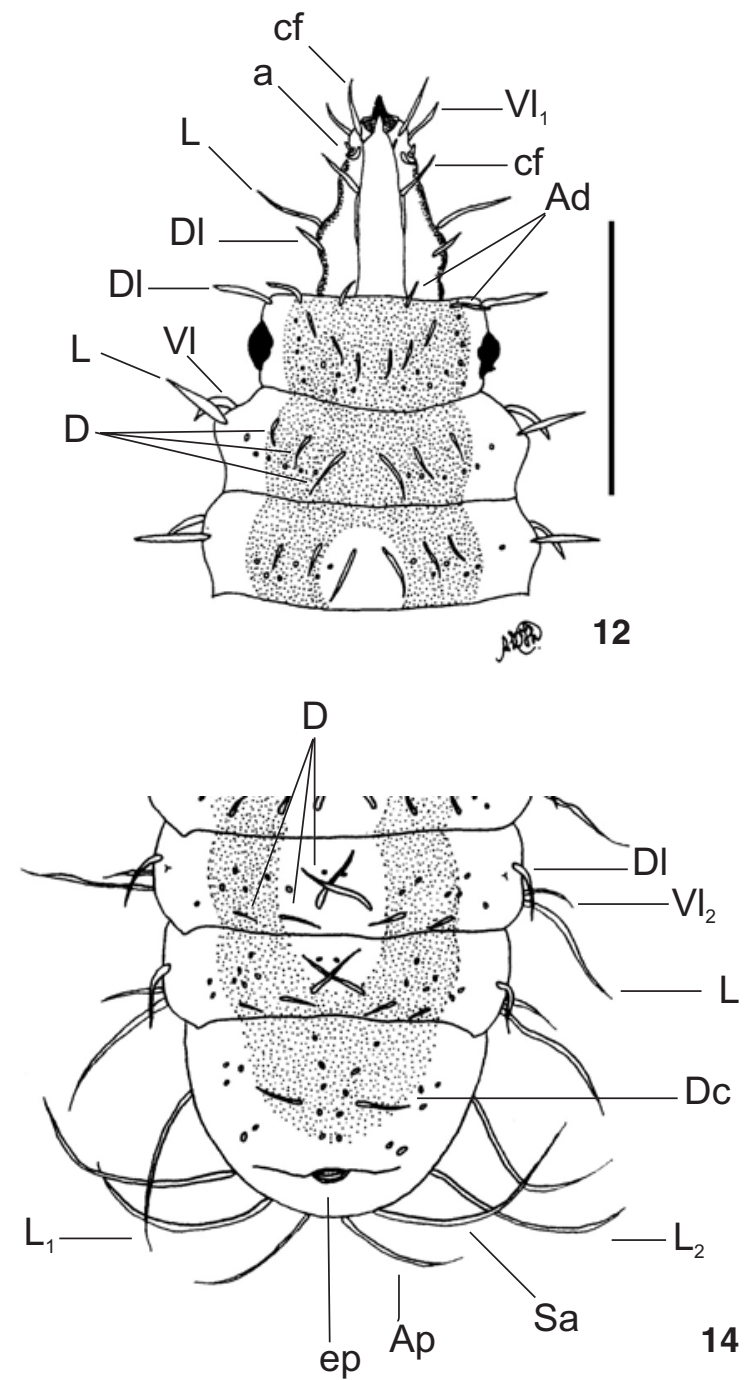

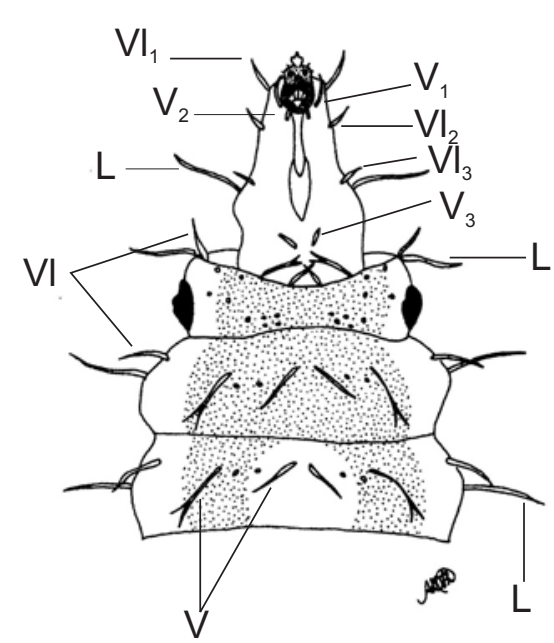

13

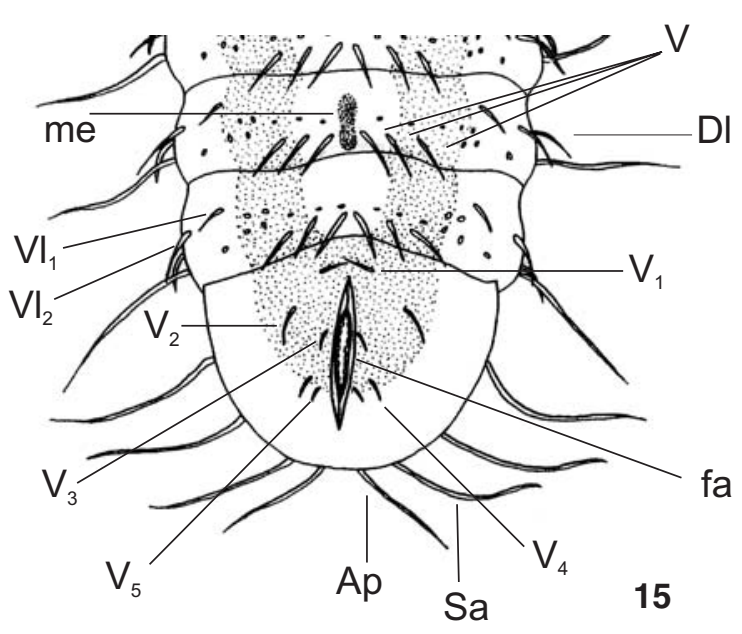

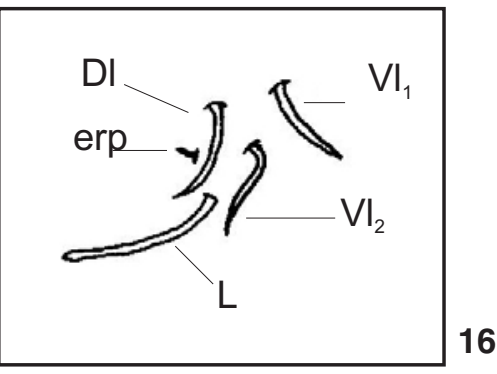

Figuras 12-16. Pedinocera longicornis: (12) cabeça: $1^{\circ}, 2^{\circ}$ e $3^{\circ}$ segmentos torácicos (vista dorsal); (13) cabeça: $1^{\circ}, 2^{\circ}$ e $3^{\circ}$ segmentos torácicos (vista ventral); (14) 6 $6^{\circ}, 7^{\circ}$ e $8^{\circ}$ segmentos abdominais (vista dorsal); (15) $6^{\circ}, 7^{\circ}$ e $8^{\circ}$ segmentos abdominais (vista ventral); (16) esquema das cerdas da região lateral dos segmentos abdominais. (a) antena; (Ad) cerdas anterodorsais; (Ap) cerdas apicais; (cf) cerdas clipeofrontais; (D) cerdas dorsais; (Dc) cerdas dorsocentrais; (DI) cerdas dorsolaterais; (ep) espiráculo posterior; (erp) espiráculo respiratório pupal; (fa) fenda anal; (L) cerdas laterais; (me) mancha esternal; ( $\mathrm{Sa}$ ) cerdas subapicais; (V) cerdas ventrais; (VI) cerdas ventrolaterais. Barra de escala $=1 \mathrm{~mm}$ (Figs $1 \mathrm{a} 4$ ). 

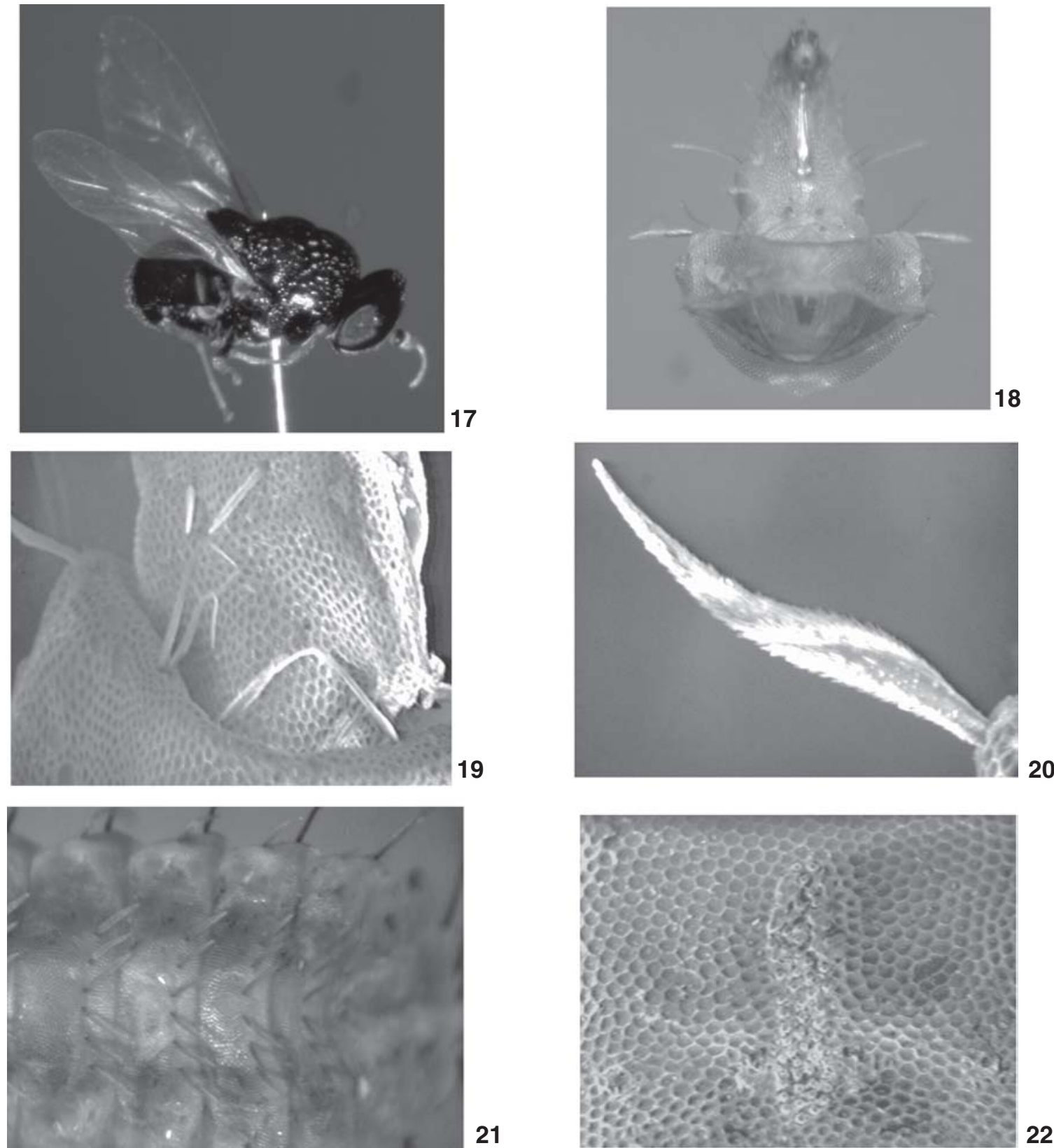

Figuras 17-22. Pedinocera longicornis: (17) adulto; (18) face ventral da cabeça (MO); (19) detalhe das cerdas ventrais bífidas do primeiro segmento torácico (MEV); (20) detalhe da cerda dorsolateral do primeiro segmento torácico (MEV); (21) detalhe das cerdas ventrais abdominais (MO); (22) detalhe da mancha esternal do $6^{\circ}$ segmento abdominal (MEV). (MEV) Microscopia eletrônica de varredura, (MO) microscopia óptica estereoscópica.

gindo até o terceiro segmento torácico e, às vezes, até o primeiro segmento abdominal.

A comparação dos pupários de $M$. crassimanus e $P$. longicornis com os pupários das sete espécies conhecidas de Pachygastrinae da Região Neotropical, resultou nas seguintes observa- ções: (1) a cabeça achatada dorsoventralmente difere de $D$. lopesi (cabeça não achatada) e de $C$. aurata (com cabeça moderadamente achatada), sendo semelhante às outras espécies; (2) os olhos pouco proeminentes diferem de C. aurata, C. argyrosticta, D. lopesi, E. major e P. modesta; (3) Em M. crassimanus o segundo

Revista Brasileira de Zoologia 23 (3): 733-739, setembro, 2006 
e terceiro segmento torácico apresentam três pares de cerdas dorsais quase tão longas quanto as laterais. Em P. longicornis as cerdas dorsais decrescem de tamanho do mais interno para o mais externo, assim como em C. aurata e C. argyrosticta, já as cerdas dorsais dos segmentos abdominais das duas espécies aqui descritas seguem o padrão de $V$. schnusei e E. major, decrescendo de tamanho do par mais interno para o mais externo; (4) em $M$. crassimanus as cerdas ventrais dos mesmos segmentos decrescem de tamanho do mais externo para o mais interno; em $P$. longicornis as cerdas ventrais são de mesmo tamanho, como em E. major, P. modesta e V. schnusei; (5) os pupários de M. crassimanus e de $P$. longicornis apresentam espiráculo respiratório pupal, em uma posição dorsolateral nos segmentos abdominais 1-6, formando um pequeno triângulo em relação a inserção das cerdas (Figs 5 e 16), esta característica também foi encontrada nas seguintes espécies: C. aurata, C. argyrosticta, E. major, P. femoralis, $P$. modesta e $V$. schnusei, sendo que nesta última o espiráculo está presente nos segmentos abdominais 1-7. Em D. lopesi os espiráculos formam uma linha reta em relação a inserção das cerdas e estão presentes nos segmentos abdominais 1-6. (6) a mancha esternal (me) do sexto segmento abdominal de Manotes crassimanus tem forma elíptica com forte dilatação na região anterior (Figs 4 e 10) semelhante a P. modesta. Em P. longicornis a mancha esternal possui semelhanças com a de C. argyrosticta, $D$. lopesi, E. major e $V$. schnusei (Figs 15 e 22). Nas demais espécies, a mancha esternal do sexto segmento possui formas elípticas variadas com ou sem estreitamento mediano.

Eupachygaster alexanderi e $Z$. stoichoides não apresentam descrições com detalhes comparáveis com M. crassimanus e $P$. longicornis, por isso não foram incluídas nas comparações acima.

\section{AGRADECIMENTOS}

Ao Centro de Adestramento da Ilha da Marambaia (CADIM - Marinha do Brasil); Fábio B. Pitombo (UFF); Geraldo Baeta (Embrapa-Agrobiologa); Karina I. de S. Marques, Luiza P. D'Almeida e Marli M. de O. Sant'Anna (ALFL e RRS). À Fundação de Amparo à Pesquisa do Estado do Rio de Janeiro - FAPERJ, processo 171.408/2002) (RDX). Ao Conselho Nacional de Desenvolvimento Científico e Tecnológico - CNPq, processo 300265/96-4 (JRPL)

\section{REFERÊNCIAS BIBLIOGRÁFICAS}

Blanchard, E.E. 1922. Apuntes sobre dos dipteros argentinos. Physis, Buenos Aires, 6: 319-323.

BRINDLE, A. 1963. Terrestrial Diptera Larvae. Entomologist's
Record, West Wickham, 75: 47-62.

JAMES, M.T. 1965. Contributions to our knowledge of the Neartic Pachygasterinae (Diptera: Stratiomyidae). Annals of the Entomological Society of America, College Park, 58 (6): 902-908

JAMES, M.T. 1973. A Catalogue of the Diptera of the Americas South of the United States. 26. Family Stratiomyidae. São Paulo, Museu de Zoologia da Universidade de São Paulo, 95p.

JAMES, M.T. 1981. Stratiomyidae, p. 497-511. In: J.F. McAlpine; B.V. Peterson; G.E. Shewell; H.J. Teskey; J.R. Vockroth \& D.M. Wood (Eds). Manual of Neartic Diptera. Ottawa, Research Branch Agriculture Canada, Monograph \#27, Vol. 1, VI+672p.

McFadden, M.W. 1967. Soldier fly larvae in America North of Mexico. Proceedings of the United States National Museum, Washington, 121 (3569): 1-72.

Pujol-Luz, J.R \& R. DE Xerez. 1999. The larva of Chalcidomorphima aurata Enderlein 1914 (Diptera: Stratiomyidae) from Ilha da Marambaia, Rio de Janeiro, Brasil. Proceedings of the Entomological Society of Washington, Washington, 101 (2): 295-299.

RozkošnÝ, R. 1982. A Biosystematic Study of the European Stratiomyidae (Diptera). Séries Entomológica, London, 21: VIII+1-401.

Rozkošný, R. \& D. Kovac. 1994. Adults and larvae of two Ptecticus Loew from Peninsular Malaysia (Diptera, Stratiomyidae). Entomologica, Milan, 137: 75-86.

Woodley, N.E. 2001. A world catalogue of the Stratiomyidae (Insecta: Diptera). Myia. The International Journal of the North Americam Dipterists' Society, Washington, 11: VII+1-473.

Xerez, R. De \& J. R. Pujol-Luz. 2001. Description of the larva of Vittiger schnusei Kertèsz, 1909 (Diptera: Stratiomyidae) from Ilha da Marambaia, Rio de Janeiro, Brazil. Studia Dipterologica, Halle, 8: 337-341.

Xerez, R. de; J.R. Pujol-Luz \& G.G. Viana. 2002. Descrição da larva de Cosmariomyia argyrosticta Kertèsz e do pupário de Dactylodeictes lopesi Lindner (Diptera, Stratiomyidae). Revista Brasileira de Zoologia, Curitiba, 19 (3): 747-755.

Xerez, R. DE; J.R. Pujol-Luz \& G.G. Viana. 2003a. Descrição da larva de Popanomyia femoralis Kertèsz, 1909 e do pupário de Engicerus major Lindner, 1964 (Diptera, Stratiomyidae). Revista Brasileira de Entomologia, Curitiba, 47 (3): 403-408.

Xerez, R. de; J.R. Pujol-Luz \& G.G. Viana. 2003b. Description of the larva of Psephiocera modesta (Lindner, 1949) (Diptera: Staratiomyidae: Pachigastrinae). Studia Dipterologica, Halle, 10: 189-193.

Recebido em 15.IX.2005; aceito em 23.VIII.2006. 